\title{
Microtubule inhibition causes epidermal growth factor receptor inactivation in oesophageal cancer cells
}

\author{
XUPING WU $^{1,4}$, LINDA SOOMAN ${ }^{1}$, JOHAN LENNARTSSON ${ }^{2}$, STEFAN BERGSTRÖM ${ }^{1}$, \\ MICHAEL BERGQVIST $^{1}$, JOACHIM GULLBO ${ }^{3}$ and SIMON EKMAN $^{1}$
}

\begin{abstract}
${ }^{1}$ Section of Oncology, Department of Radiology, Oncology and Radiation Sciences, Uppsala University, SE-75185 Uppsala;
${ }^{2}$ Ludwig Institute for Cancer Research, Uppsala University, SE-75124 Uppsala; ${ }^{3}$ Department of Medical Sciences, Division of Clinical Pharmacology, Uppsala University, SE-75185 Uppsala, Sweden; ${ }^{4}$ The Second Hospital of Nanjing affiliated to Southeast University, 210003 Nanjing, P.R. China
\end{abstract}

Received September 18, 2012; Accepted November 2, 2012

DOI: $10.3892 /$ ijo.2012.1710

\begin{abstract}
Drugs that interfere with microtubule function can prevent cells from mitosis and may cause cell cycle arrest or apoptosis. Various microtubule targeting agents, both stabilizers and inhibitors, are used in a clinical setting to treat cancer. In the current study, we investigated the sensitivity of oesophageal cancer cells to different microtubule targeting agents. The current study demonstrated that different microtubule targeting agents disrupted the microtubule network and inhibited survival of oesophageal cancer cells in a dosedependent manner. Interestingly, an additional cellular effect with inhibition of tyrosine phosphorylation of the EGFR and subsequent downregulation of EGFR-induced signalling was also observed, suggesting an additional mechanism of action for microtubule destabilising agents. A tyrosine phosphatase inhibitor, sodium orthovanadate, could reverse the EGFR dephosphorylation effects induced by microtubule targeting agents. The EGFR dephosphorylation could be reversed by a tyrosine phosphatase inhibitor, indicating that disruption of the microtubule network may lead to activation of a protein tyrosine phosphatase (PTP) that can regulate EGFR phosphorylation and activation, an effect of potential clinical relevance for combination therapies in patients.
\end{abstract}

\section{Introduction}

The cytoskeleton is comprised of actin filaments, intermediate filaments and microtubules. Microtubules are dynamic structures that are important for a range of cellular functions, such as intracellular trafficking, cell movement and division,

Correspondence to: Dr Xuping Wu, The Second Hospital of Nanjing affiliated to Southeast University, 1-1 Zhongfu Road, 210003 Nanjing, P.R. China

E-mail: xuping_wu@yahoo.com

Key words: microtubule targeting agents, epidermal growth factor, tyrosine phosphatase, oesophageal cancer cells where they are involved in chromosome segregation. $\alpha$ - and $\beta$-tubulin heterodimers polymerise into a hollow tube denoted microtubule $(1,2)$. The stability of the microtubule is regulated through GTP-hydrolysis of $\beta$-tubulin; binding of GTP allows polymerisation, but within the microtubule GTP can be hydrolysed to GDP $(1,2)$. Microtubules constantly polymerise and depolymerise, a process termed dynamic instability. Since microtubules play an essential role in chromosome segregation, drugs that interfere with microtubule function prevent the cell from mitosis. A number of microtubule targeting drugs are used in the clinic to treat human cancers, including oesophageal cancer. Examples are the vinca alkaloids that destabilise microtubules by binding close to the GTP-binding region in $\beta$-tubulin (3), and the taxanes which bind to polymerised microtubules and stabilise the GDP-bound form of $\beta$-tubulin by forcing them into a configuration resembling the GTP-bound state (4).

Growth factors and their receptors have been shown to be of pivotal significance for the occurrence and development of cancer. The human epidermal growth factor receptor (HER) family is comprised of four members, i.e. EGFR (HER1, ErbB1), HER2 (ErbB2, Neu), HER3 (ErbB3) and HER4 (ErbB4) (5). These receptors are tyrosine kinases that are activated by ligand-induced dimerisation. There are several ligands for the receptors in the HER family, and these have different binding specificities, resulting in formation of homo- or heterodimeric receptor complexes. HER family members are commonly activated in human cancer cells by different mechanisms including autocrine stimulation, mutations or overexpression (6). Dysregulated and improper receptor activation leads to induction of signals that promote proliferation, survival, migration and angiogenesis, events that are all central for tumour development and progression. Over-activated EGFR is recognised as an important mechanism in several types of cancer, including colorectal cancer (7), head and neck cancer (8), and non-small cell lung cancer (9) and has become a target of interest in the treatment of these tumours.

There are data indicating possible interactions between EGFR and the microtubule system. Gao et al demonstrated that histone deacetylase 6 (HDAC6), a microtubule-associated deacetylase, associates with the endosomal compartments 
and controls EGFR trafficking and degradation (10). This is consistent with data from Deribe et al showing that HDAC6 negatively regulates EGFR endocytosis and degradation by controlling the acetylation status of $\alpha$-tubulin and subsequently receptor trafficking along microtubules (11).

Oesophageal carcinoma is the seventh most common cause of cancer-related death in the Western world (12). The standard treatment for localised oesophageal carcinoma includes a combination of radiation and chemotherapy, sometimes followed by surgery. Preoperative chemoradiotherapy or chemotherapy (13) has been demonstrated to give a significant survival benefit. However, patients with advanced metastatic disease that are treated with palliative chemotherapy have a poor prognosis with a median survival time of less than one year. The 5-year survival rate of all diagnosed patients is only around $15 \%$. Thus, there is an urgent need to improve current therapies. In oesophageal carcinoma patients, EGFR has been reported to be commonly overexpressed (14) and the overexpression is correlated to lymph node metastasis, vascular invasion and shorter survival (15-17). The EGF and TGF ligands function as mitogens for oesophageal tumour cells (18) and activation of EGFR signalling has been implicated in metastasis via modulation of cell adhesion, angiogenesis, invasion and migration.

In the current study we have investigated the possible interaction of anti-microtubule drugs and the EGFR signalling system in human oesophageal cancer cells. Treatment with the drugs led to inhibition of proliferation of the cells. Additionally, microtubule destabilising agents were also shown to inhibit EGFR phosphorylation. These effects could be inhibited by simultaneous addition of the protein tyrosine phosphatase inhibitor sodium orthovanadate, suggesting that disruption of the microtubule network leads to release or activation of a tyrosine phosphatase. This study shows that microtubule targeting drugs have other effects beyond interfering with the mitotic spindle.

\section{Materials and methods}

Cell culturing and counting. The human ESCC (oesophageal squamous cell carcinoma) cell lines Kyse30, Kyse70, Kyse140, Kyse150, Kyse180, Kyse410, Kyse450, Kyse510 and Kyse520 (Deutsche Sammlung von Mikroorganismen und Zellkulturen $\mathrm{GmbH}$, Braunschweig, Germany) were cultivated in RPMI-1640 medium, supplemented with $10 \%$ fetal bovine serum (FBS), $2 \mathrm{mM}$ L-glutamine, 50 units of penicillin and $50 \mu \mathrm{g}$ streptomycin/ml (Sigma-Aldrich, St. Louis, MO, USA). Cells were split twice a week by incubation in $13-20 \mu \mathrm{l} / \mathrm{cm}^{2}$ 5X Trypsin-EDTA Solution (Sigma-Aldrich) in $37^{\circ} \mathrm{C}$ until detachment from the surface. Cells were used for further experiments when 70-90\% confluent. Cell counting was performed using a Coulter ${ }^{\circledR} \mathrm{Z} 2$ Particle count and size analyser.

Cell growth analysis using resazurin assay. Kyse70 and Kyse140 cells were seeded in 96-well plates at a concentration of 5,000 cells per well and were allowed to grow overnight. Docetaxel, podophyllotoxin (PPT) and vincristine are from SigmaAldrich. Five or seven different concentrations of docetaxel, podophyllotoxin (PPT) or vincristine were then added to the cell medium. After 24 and $48 \mathrm{~h}$, resazurin (Alamar Blue, Sigma-Aldrich) was added at a concentration of $440 \mu \mathrm{M}$ to each well followed by incubation in the dark for $1 \mathrm{~h}$ at room temperature. Wells containing only culture medium served as blank. The analysis of fluorescence (560EX $\mathrm{nm} / 590 \mathrm{EM} \mathrm{nm})$ using a Wallac Victor Multilabel Counter (Wallac, Turku, Finland) was followed by calculations of relative numbers of viable cells expressed as percentages of untreated cells. Resazurin detects cell viability by converting the reagent to a fluorescent indicator in response to metabolically active cells $(19,20)$. The resazurin assay is quantitative with respect to time and dose, and separate experiments showed a linear correlation between the number of viable cells and the emitted light (data not shown). Each experiment was performed three times.

Microtubule staining. Kyse70 and Kyse140 cells were grown on coverslips overnight and then treated with $5 \mu \mathrm{M}$ of either docetaxel, PPT or vincristine for $24 \mathrm{~h}$. Cells were fixed in $2 \%$ formaldehyde and permeabilised with $0.2 \%$ Triton X-100. Coverslips were blocked with $10 \mathrm{mMglycine}$ at room temperature for $1 \mathrm{~h}$, incubated with primary mouse anti- $\alpha$-tubulin antibody (Sigma-Aldrich), followed by incubation with a secondary polyclonal goat anti-mouse antibody labelled with FITC (Dako, Glostrup, Denmark). The nuclei were stained with DAPI. Coverslips were mounted on object slides using Fluoromount-G (Southern Biotechnology Associates, Birmingham, AL, USA). Microtubule staining was visualised using a Zeiss immunofluorescence microscope at $\mathrm{x} 40$ magnification.

Immunoprecipitation and western blot analysis. Subconfluent Kyse70 and Kyse140 cells were treated with different concentrations $(0.5,5$ and $10 \mu \mathrm{M})$ of PPT, vincristine or docetaxel for 24 and $48 \mathrm{~h}$ in starvation medium containing $0.1 \%$ FBS. Subsequently, cells were stimulated with $100 \mathrm{ng} / \mathrm{ml}$ EGF (Chemicon, Temecula, CA, USA) or IGF-1 (R\&D Systems, Minneapolis, MN, USA) for 5 min and washed with ice-cold phosphate-buffered saline before lysis. Cell lysates were prepared according to Lennartsson et al (21). Briefly, total protein concentration was determined using the BCA Protein Assay Kit (Pierce, Rockford, IL, USA). Total cell lysate (TCL) were submitted to SDS-polyacrylamide gel electrophoresis (SDS-PAGE). For immunoprecipitation, antibodies against IGF-1R $\beta$ were added to each lysate at a concentration of $1 \mu \mathrm{g} / \mathrm{ml}$. Protein A-Sepharose was added in order to collect immunocomplexes. After washing of the beads, samples were boiled in reducing sample buffer and subjected to SDS-PAGE. Separated proteins were electrotransferred to polyvinylidene fluoride (PVDF) membranes (Millipore, Billerica, MA, USA), membranes blocked using 5\% BSA, and then incubated with primary antibody overnight at $4^{\circ} \mathrm{C}$. Antibodies used were anti-EGFR, anti-IGF-1R $\beta$ and anti-Akt1/2 rabbit polyclonal antibodies (Santa Cruz Biotechnology, Santa Cruz, CA, USA), anti-phosphotyrosine mouse monoclonal antibodies PY99 (Santa Cruz Biotechnology), phospho-specific anti-Erk and phospho-specific anti-Akt antibodies (Cell Signalling Technology, Beverly, MA, USA), anti-PTP1B mouse antibodies (BD Biosciences, San Jose, CA, USA), anti- $\beta$-actin mouse monoclonal antibodies (Sigma-Aldrich) and anti-Erk2 rabbit serum (Ludwig Institute for Cancer Research, Uppsala, Sweden). Anti-PTPE rabbit serum was from Dr A. Elson (The Weizmann Institute of Science, Israel). EGF was from Chemicon and IGF-1 from R\&D Systems. After washing, membranes were 

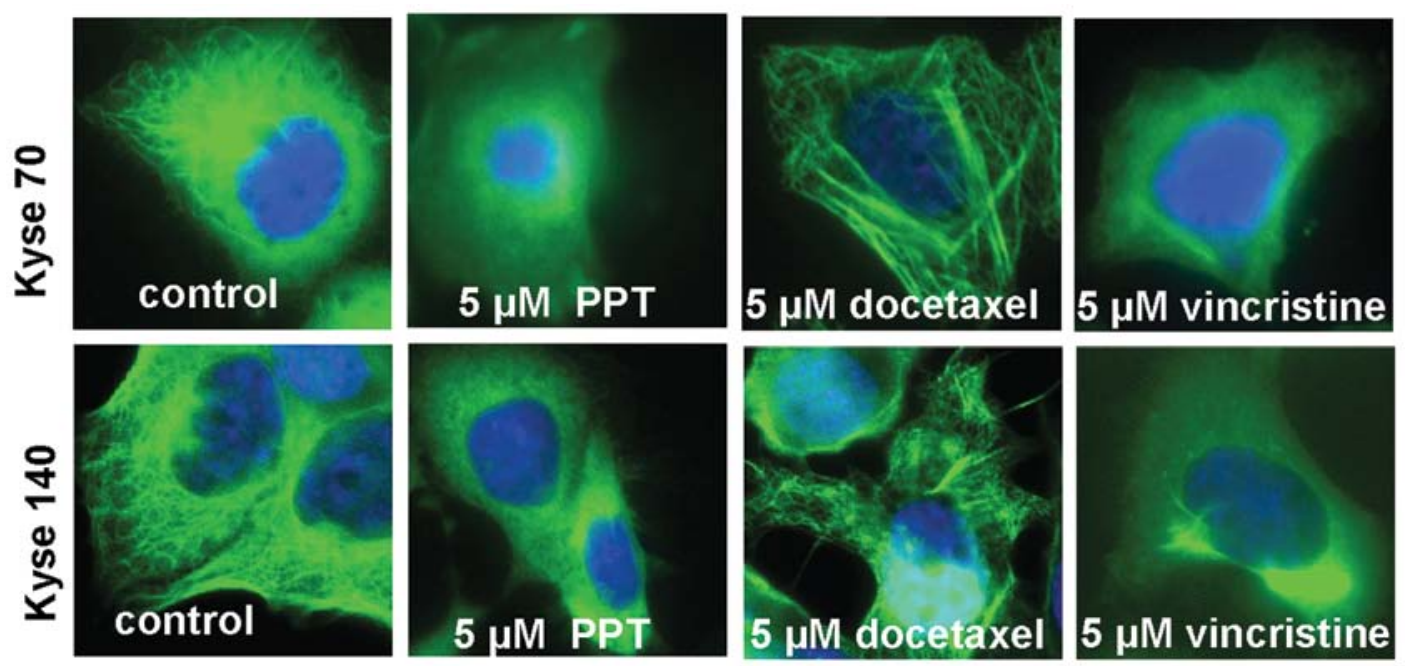

Figure 1. The effect of microtubule targeting drugs on microtubule network in oesophageal carcinoma cells. Kyse70 and Kyse140 cells were grown on coverslips and treated with different concentrations of microtubule targeting drugs PPT, docetaxel or vincristine. Cells were fixed with $2 \%$ formaldehyde and microtubules (green) analysed by fluorescence microscopy. The figure is a representative of three repeats.
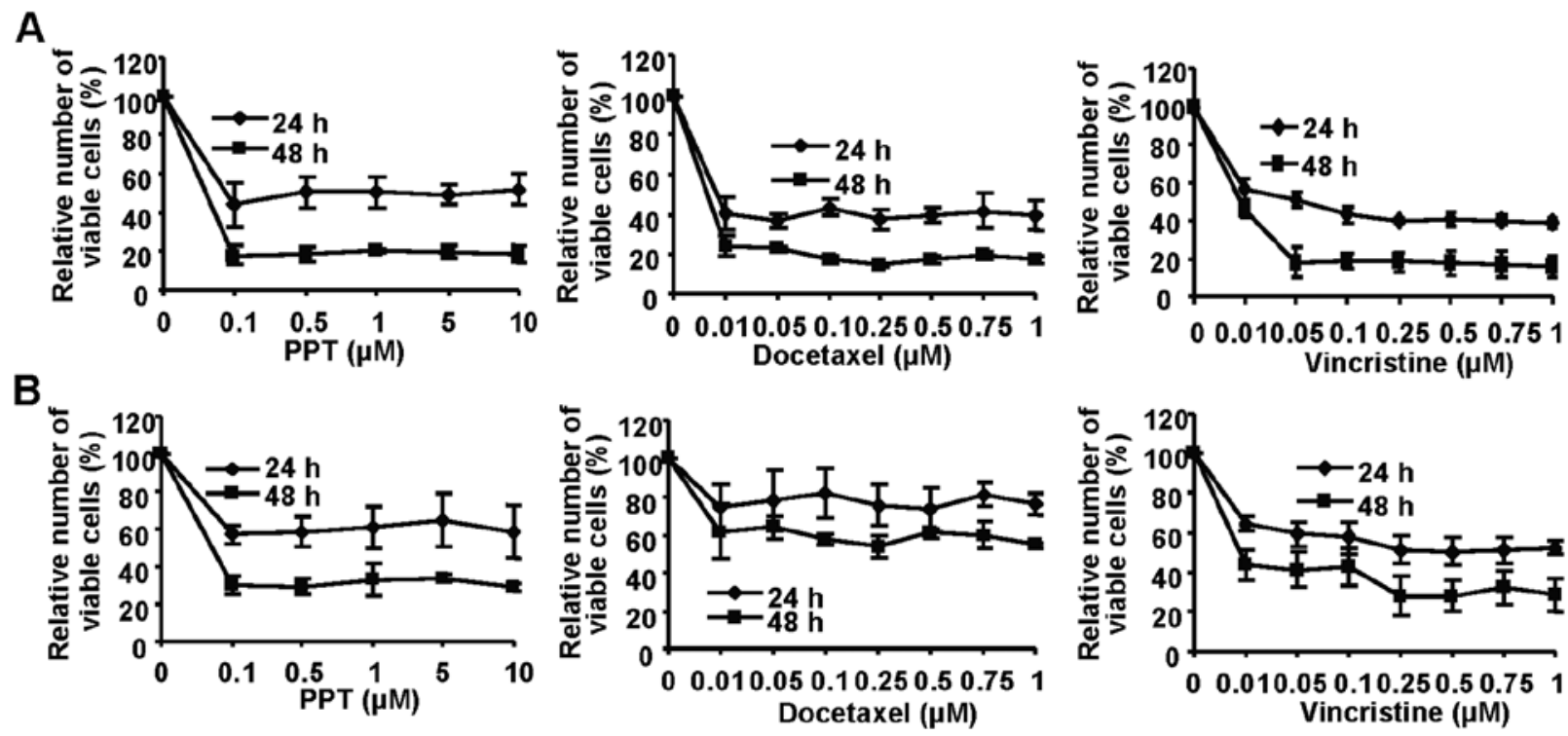

Figure 2. Effects of microtubule targeting agents on proliferation in (A) Kyse70 and (B) Kyse140 cells. ESCC cells were treated with PPT, docetaxel or vincristine at indicated concentrations for 24 and $48 \mathrm{~h}$, and cell survival analysed using the resazurin assay. Kyse70 and Kyse140 cells were seeded into 96-well plates at a density of 5,000 cells/well. The experiments were done in triplicates and error bars represent $\pm \mathrm{SD}$.

incubated with horseradish peroxidase-conjugated anti-mouse or anti-rabbit IgG antibodies (Amersham Biosciences, Uppsala, Sweden) and proteins visualised using ECL western blotting detection systems (Roche Applied Science, Indianapolis, IN, USA) on a cooled charge-coupled device (CCD) camera (Bio-Rad Life Science, Hercules, CA, USA). The images were analysed using the software Quantity One.

siRNA interference. siRNA was employed to knockdown PTPE and PTP1B expression. Anti-PTPE siRNA targets sequence: GCGAACAGGUACAUUCAUA; anti-PTP1B siRNA targets sequence: GGAGAAAGGUUCGUUAAAA; non-targeting siRNA was used as a control (target sequence CGTACGCGGA
ATACTTCGA). To downregulate PTP1B and PTPe expression, different concentrations of siRNA for anti-PTP1B and anti-PTPE were incubated with Kyse 70 and Kyse 140 cells for $48 \mathrm{~h}$. Levels of knockdown were tested after measuring protein levels by immunoblotting. Meanwhile, different concentrations of microtubule targeting drugs were added to the cell cultures for $24 \mathrm{~h}$.

Sodium orthovanadate treatment. Subconfluent Kyse70 and Kyse140 cells were treated with $10 \mu \mathrm{M}$ PPT, vincristine or docetaxel for $24 \mathrm{~h}$. Before cell lysis, sodium orthovanadate $\left(\mathrm{Na}_{3} \mathrm{VO}_{4}\right)$ was added in the medium at a concentration of $1 \mathrm{mM}$ for $1 \mathrm{~h}$, followed by stimulation with $100 \mathrm{ng} / \mathrm{ml} \mathrm{EGF}$ for $5 \mathrm{~min}$. Total cell lysates were used for immunoblotting analysis. 


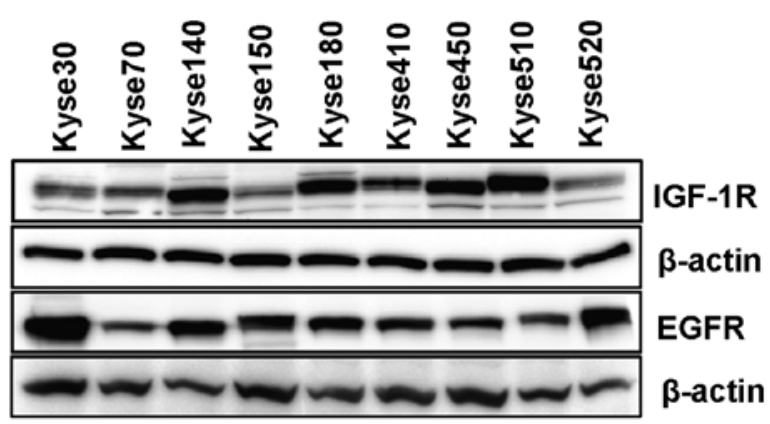

Figure 3. EGFR and IGF-1R expression in 9 oesophageal carcinoma cells. Western blotting was done to detect EGFR and IGF-1R expression. $\beta$-actin was used as a loading control. A representative of three blots was shown.

\section{Results}

Podophyllotoxin (PPT), vincristine and docetaxel interact with the microtubule network and affect survival of ESCC cells. After 24-h treatment, the microtubule destabilising drugs PPT $(5 \mu \mathrm{M})$ and vincristine $(5 \mu \mathrm{M})$ disrupted the microtubule network while the microtubule stabilising drug docetaxel $(5 \mu \mathrm{M})$ stabilised the network (Fig. 1) in oesophageal cancer cells. After 24-h drug treatment, we did not observe nuclear pyknosis and massive apoptotic bodies in either Kyse70 or Kyse140 cells under microscope following DAPI staining. Treatment with PPT, docetaxel and vincristine in Kyse70 and Kyse140 cells resulted in time-dependent plateau-shaped doseresponse curves in a proliferation assay, consistent with tubulin interaction and cell cycle arrest. Maximal inhibition $(>80 \%)$ of Kyse70 (Fig. 2A) was achieved at low concentrations $(0.1 \mu \mathrm{M}$ PPT, $0.1 \mu \mathrm{M}$ docetaxel and $0.05 \mu \mathrm{M}$ vincristine at $48 \mathrm{~h}$ ), while Kyse140 cells appeared slightly less sensitive (Fig. 2B).
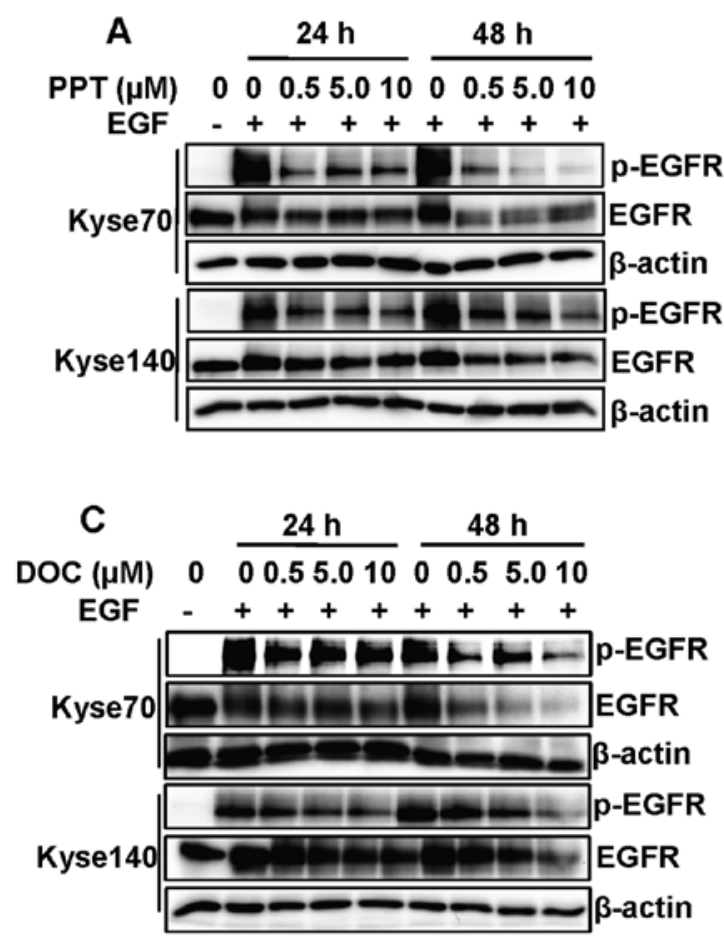

Microtubule destabilising drugs decrease EGFR phosphorylation and downstream signalling in ESCC cells. It has been reported that EGFR is commonly overexpressed in oesophageal carcinoma (14). Variable but detectable levels of EGFR were observed in all 9 oesophageal carcinoma cell lines used in this study (Fig. 3). The possible interactions between EGFR and microtubules were investigated by treatment of Kyse70 and Kyse140 cells with increasing concentrations of microtubule targeting drugs for 24 or $48 \mathrm{~h}$, followed by $5 \mathrm{~min}$ EGF stimulation. The concentrations used in this study are comparable to or slightly higher than the clinically achievable peak plasma concentrations in patients $(22,23)$. The microtubule destabilising agents PPT (Fig. 4A) and vincristine (Fig. 4B) inhibited EGFR tyrosine phosphorylation after $24 \mathrm{~h}$ treatment at all concentration where we did not observe the inhibition of EGFR expression. However, the microtubule stabilising agent docetaxel had a minor inhibitory effect on EGFR phosphorylation after $24 \mathrm{~h}$ treatment (Fig. 4C). After $48 \mathrm{~h}$ of drug treatment with PPT, vincristine and docetaxel, a substantial inhibition of EGFR expression and phosphorylation could be observed, indicating a possible indirect effect due to the cytotoxicity of microtubule targeting drugs (Fig. 4). Dephosphorylation was observed already after $24 \mathrm{~h}$ of treatment whereas EGFR downregulation was most pronounced after $48 \mathrm{~h}$, indicating that inhibition of phosphorylation and receptor downregulation are two distinct events.

To investigate if decreased phospho-EGFR also resulted in decreased signalling downstream of EGFR, cells were treated with microtubule targeting drugs for $24 \mathrm{~h}$ thus avoiding EGFR degradation. Docetaxel, PPT and vincristine downregulated phosphorylation as well as protein levels of Akt (Fig. 5) in both Kyse70 and Kyse140 cells. Moreover, the microtubule destabilising agents PPT and vincristine inhibited phosphorylation of Erk but did not cause downregulation of total Erk protein

Figure 4. Effects of microtubule targeting drugs on EGFR. Kyse70 and Kyse140 cells were starved and then treated with indicated concentrations of (A) PPT, (B) vincristine (VCR) or (C) docetaxel (DOC) for 24 or $48 \mathrm{~h}$. After stimulation with EGF for 5 min, western blotting was done on total cell lysates for detection of phosphorylated EGFR and total EGFR levels. $\beta$-actin was used as a loading control for each blot. The experiments were repeated three times with similar results. 
levels. Docetaxel had no effect on the activation of Erk in either cell line.

Microtubule targeting drugs demonstrated no inhibition of phosphorylation or expression of IGF-1R in ESCC cells. IGF-1R, which is becoming an important target in the treatment of cancer, has been found to be significantly overexpressed in oesophageal squamous cell carcinoma tissue compared with adjacent normal tissue (24). We found that IGF-1R was expressed in all nine ESCC cell lines (Fig. 3). To test whether microtubule targeting drugs can affect IGF-1R, we treated cells with increasing concentrations of microtubule targeting drugs $(0.5,5$ and $10 \mu \mathrm{M})$ for 24 or $48 \mathrm{~h}$, followed by 5 min of IGF-1 stimulation. We were not able to observe any effect either on ligand-induced IGF-1R phosphorylation or on IGF-1R expression (Fig. 6). Thus, it appears that disruption of the microtubule system selectively inhibits EGFR function over that of IGF-1R, indicating that EGFR downregulation is not caused by general drug cytotoxicity.

Reduced EGFR phosphorylation induced by microtubule destabilising agents can be reversed by treatment with a tyrosine phosphatase inhibitor. EGFR dephosphorylation caused by microtubule destabilising agents was not correlated with protein downregulation for all drugs and cell lines. In addition, the EGFR dephosphorylation occurred before substantial receptor downregulation could be seen, suggesting that these effects are independent of each other (Fig. 4). To further explore the mechanism of EGFR dephosphorylation we treated cells for $24 \mathrm{~h}$, to avoid major EGFR downregulation, with microtubule targeting drugs in the presence or absence of the tyrosine phosphatase inhibitor sodium orthovanadate $\left(\mathrm{Na}_{3} \mathrm{VO}_{4}\right)$. As can be seen in Fig. 7, sodium orthovanadate treatment to a large extent abolished EGFR dephosphorylation. Thus, one possibility is that disruption of the microtubule network releases or activates a tyrosine phosphatase that can dephosphorylate EGFR but not IGF-1R.

PTP1B or PTPE downregulation can not reverse EGFR dephosphorylation induced by microtubule disrupting agents. The tyrosine phosphatase PTPe co-localises with microtubules in cells and its binding to tubulin can inhibit its activity; conversely disrupting microtubule structures increased PTPE activity (25). PTP1B has been reported to interact with endocytosed EGFR and promote its dephosphorylation, and this complex is disrupted by sodium othovanadate $(26,27)$. To further explore which protein tyrosine phosphatase is involved in EGFR dephosphorylation induced by microtubule disruption, RNAi was employed to downregulate PTP1B and PTPE expression in Kyse70 and Kyse140 cells as shown in Fig. 8A and B. Neither PTP1B nor PTPE downregulation could reverse the effect of the microtubule disrupting drugs on EGFR dephosphorylation (Fig. 8C and D). Furthermore, we tested if PTPE downregulation could affect the EGFR dephosphorylation induced by microtubule targeting agents in the A431 cell line which is originated from epidermoid carcinoma and known to overexpress EGFR. PPT dephosphorylated EGFR in both A431 moc (cytPTPe negative) and A431 (cytPTPE overexpressed) cells (Fig. 8E), proving also in other cells than ESCC cells that PTPE downregulation can not reverse EGFR dephosphorylation induced by microtubule targeting agents.

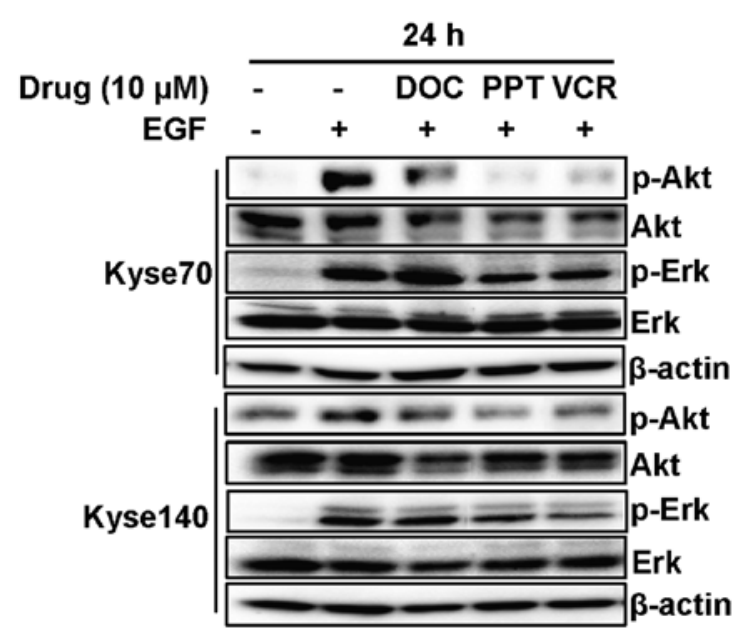

Figure 5. Effects of microtubule targeting agents on EGFR downstream signalling. Kyse70 and Kyse140 cells were starved and then treated with $10 \mu \mathrm{M}$ of PPT, vincristine (VCR) or docetaxel (DOC) for $24 \mathrm{~h}$. After stimulation with EGF for $5 \mathrm{~min}$, western blotting was done on total cell lysates for detection of phospho-Akt, Akt, phospho-Erk and Erk levels. $\beta$-actin was used as a loading control. The figure is a representative of three repeats.

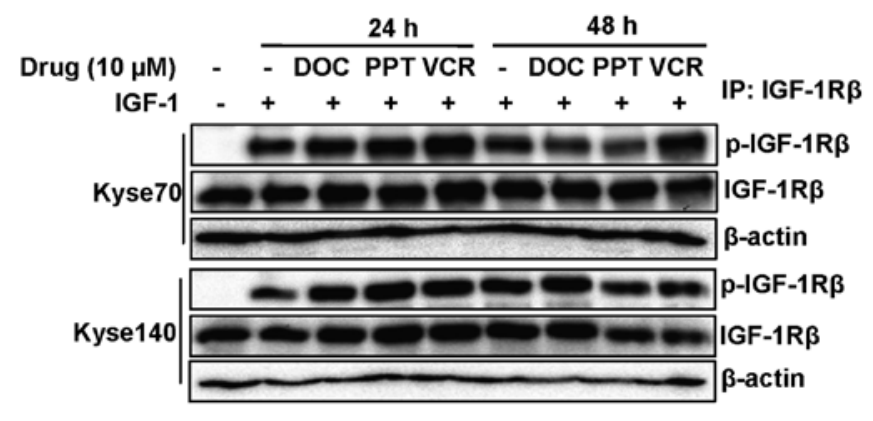

Figure 6. Effects of microtubule targeting agents on IGF-1R. Kyse70 and Kyse140 cells were starved and treated with $10 \mu \mathrm{M}$ docetaxel (DOC), PPT or vincristine (VCR) for 24 or $48 \mathrm{~h}$. After stimulation with IGF-1 for $5 \mathrm{~min}$, IGF-1R was immunoprecipitated and western blotting was done for detection of phosphorylated IGF-1R. $\beta$-actin was used as a loading control. The figure is a representative of three repeats.

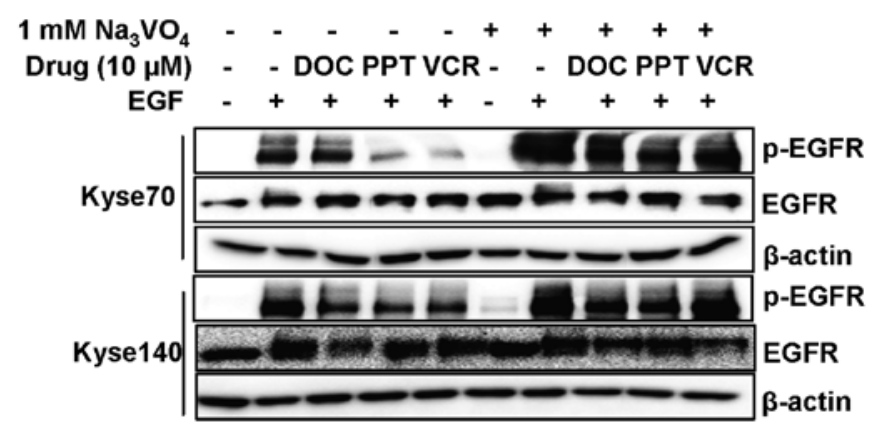

Figure 7. The effect of tyrosine phosphatase inhibition on reduced EGFR phosphorylation induced by microtubule destabilising agents. Kyse70 and Kyse140 cells were grown to sub-confluency, starved and treated with $10 \mu \mathrm{M}$ docetaxel (DOC), PPT or vincristine (VCR) for $24 \mathrm{~h}$. Before stimulation with EGF, cells were incubated with $1 \mathrm{mM}$ sodium orthovanadate for $1 \mathrm{~h}$. Western blotting was done on total cell lysates to detect phosphorylated EGFR and total EGFR levels. $\beta$-actin was used as a loading control for each blot. The experiments were repeated three times, a representative result is shown. 

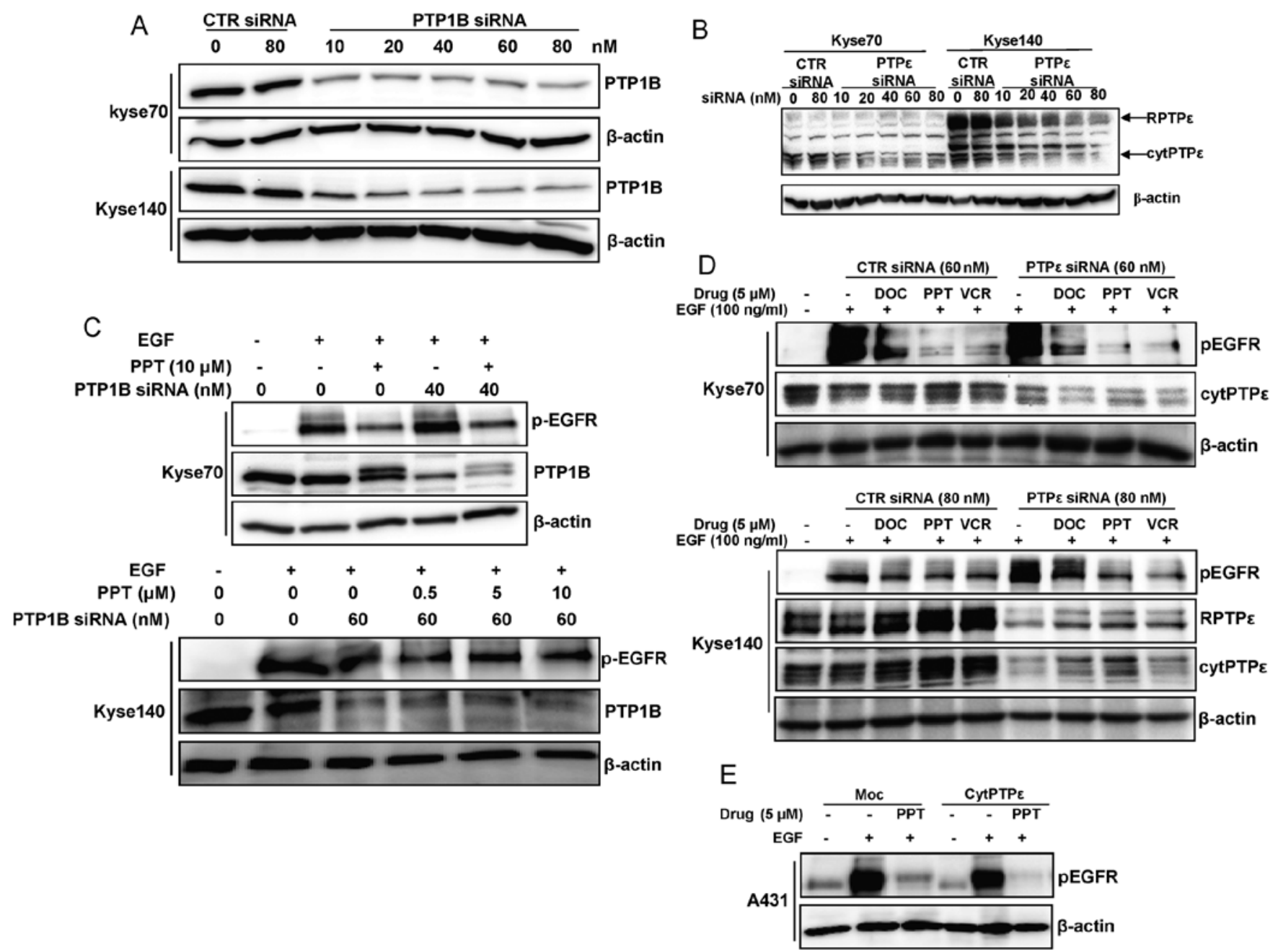

Figure 8. PTP1B or PTPE downregulation could not reverse EGFR dephosphorylation induced by microtubule disrupting agents. (A and B) The anti-PTP $\varepsilon$ antibody

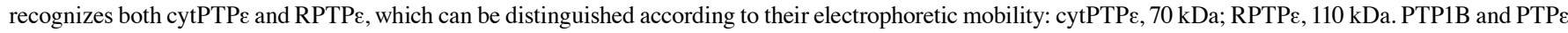
was downregulated by specific siRNA transfected using siLentFect ${ }^{\mathrm{TM}}$ from Bio-Rad in Kyse70 and Kyse140 cells. For each experiment, non-targeting siRNA was used as a control. $\beta$-actin was used as a loading control for each blot. (C) Kyse70 and Kyse140 cells were grown to sub-confluency, starved and treated with $10 \mu \mathrm{M}$ PPT for $24 \mathrm{~h}$ to avoid EGFR degradation. Meanwhile, specific siRNA was added to downregulate PTP1B expression. After stimulation with EGF, western blotting was performed on total cell lysates to detect phosphorylated EGFR as well as PTP1B. $\beta$-actin was used as a loading control for each blot. (D) Kyse70 and Kyse140 cells were grown to sub-confluency, starved and treated with $5 \mu \mathrm{M}$ vincristine (VCR), docetaxel (DOC) or PPT for $24 \mathrm{~h}$ to avoid EGFR degradation. Meanwhile, PTPE expression was downregulated using siRNA. After stimulation with EGF, western blotting was performed on total cell lysates to detect phosphorylated EGFR

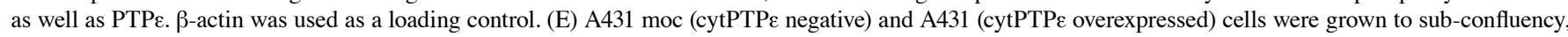
starved and treated with $5 \mu \mathrm{M}$ PPT for $24 \mathrm{~h}$ to avoid EGFR degradation. After stimulation with EGF, western blotting was performed on total cell lysates to detect phosphorylated EGFR. Each experiment has been repeated at least three times. Non-targeting siRNA was used in all experiments as a control.

\section{Discussion}

Microtubules are involved in various cellular functions, including cell adhesion, movement, replication and division. Microtubule inhibition can interfere with chromosome segregation during mitosis and disrupt cell signalling, hence promoting cell cycle arrest and cell death $(28,29)$. In the present study, we have investigated the cytotoxic effects of the microtubule targeting drugs docetaxel, vincristine, and PPT in oesophageal carcinoma cell lines. As expected, microtubule targeting drugs disrupted the microtubule network and inhibited cell survival in oesophageal carcinoma cells. Surprisingly, we also found that disruption of the microtubule network was associated with dephosphorylation of EGFR and subsequent reduced activation of Akt and Erk. Co-treatment with a tyrosine phosphatase inhibitor diminished this effect, suggesting that disruption of the microtubule network leads to exposure of EGFR to an active tyrosine phosphatase. Neither 24 nor $48 \mathrm{~h}$ of drug treatment had any effect on IGF-1R phosphorylation or stability, suggesting some degree of receptor selectivity and that the EGFR downregulation is not due to general toxicity.

In both Kyse70 and Kyse 140 cells, phosphorylation of Akt was inhibited by docetaxel, vincristine and PPT. However, the degradation of total Akt protein after drug treatment may partially explain the dephosphorylation of Akt. Compared to Akt, Erk protein levels were not affected by drug treatment, while phosphorylation of Erk was inhibited by vincristine and PPT but not docetaxel in both Kyse70 and Kyse140 cells. This suggests that Akt protein levels may be more easily affected by a general drug response and that microtubule destabilisa- 
tion, but not stabilisation, affects downstream signalling of EGFR. The observed dephosphorylation of EGFR could be reversed by a tyrosine phosphatase inhibitor, suggesting that a tyrosine phosphatase is activated following microtubule disruption. It is possible that microtubule destabilising agents activate a phosphatase that is selective for EGFR over IGF-1R in ESCC cells. Protein tyrosine phosphatases (PTPs) strictly control receptor tyrosine kinase (RTK) phosphorylation and downstream signalling. Several PTPs have been reported to dephosphorylate tyrosine residues of EGFR and regulate signalling, including T-cell PTP (TCPTP), Src homology phosphotyrosine phosphatase 1 and 2 (SHP1 and 2), PTP1B, PTPN9, density-enhanced phosphatase-1 (DEP-1), RPTPo and RPTPк (30-35). So far only PTPE and PTP1B have been reported to interact with the microtubule system (25-27). However, using siRNA to downregulate PTP1B and PTPE expression in ESCC cells, we found no evidence supporting that PTP1B or PTPE downregulation could influence the effect of the microtubule disrupting drugs on EGFR dephosphorylation (Fig. 8C and D). Elucidating which PTP(s) is important for regulation of EGFR phosphorylation in ESCC cells following disruption of the microtubule network is subject for future studies.

The additional mechanism of action of tubulin inhibitors on EGFR signalling suggested in the present work may have clinical impact on the selection of drug combinations for the treatment of oesophageal cancer as well as other cancer types. Several clinical studies involving EGFR targeted therapies in oesophageal cancer have been performed, including the antibody cetuximab as well as the tyrosine kinase inhibitors erlotinib (Tarceva ${ }^{\circledR}$ ) and gefitinib (Iressa ${ }^{\circledR}$ ). Although not yet a standard of care, the results from these studies suggest that treatment with EGFR targeted therapies, alone or in combination with chemotherapy and/or radiotherapy, is feasible with promising clinical activity $(36,37)$. Ongoing and future clinical trials involving EGFR targeted therapies and anti-tubulin acting chemotherapy in combination is recommended to consider the potential interactions between these treatments, both with respect to clinical efficacy of the treatment and to the selection of appropriate biomarkers.

We demonstrated that microtubule targeting drugs inhibited the survival of oesophageal cancer cells involving a reduction of tyrosine phosphorylation and activation of EGFR, and that this effect is reversible by inhibition of tyrosine phosphatases using sodium orthovanadate. Thus, we propose that in addition to the previously described mechanisms of action for microtubule disrupting chemotherapeutics, these drugs may also lead to EGFR dephosphorylation and downregulation of EGFR-induced signalling. These findings may have a clinical impact on the selection of chemotherapeutic drug combinations for the treatment of oesophageal cancer as well as other cancer types.

\section{Acknowledgements}

The authors are grateful to Dr Markus Dagnell at Cancer Center, Karolinska Institutet, for providing A431 moc (cytPTPe negative) and A431 (cytPTPE overexpressed) cells. We also thank Dr Ari Elson from Weizmann Institute of Science for providing anti-PTPE antibody.

\section{References}

1. Li H, DeRosier DJ, Nicholson WV, Nogales E and Downing KH: Microtubule structure at 8 A resolution. Structure 10: 1317-1328, 2002.

2. Mitchison T and Kirschner M: Dynamic instability of microtubule growth. Nature 312: 237-242, 1984

3. Lobert S, Vulevic B and Correia JJ: Interaction of vinca alkaloids with tubulin: a comparison of vinblastine, vincristine, and vinorelbine. Biochemistry 35: 6806-6814, 1996.

4. Burkhart CA, Berman JW, Swindell CS and Horwitz SB: Relationship between the structure of taxol and other taxanes on induction of tumor necrosis factor-alpha gene expression and cytotoxicity. Cancer Res 54: 5779-5782, 1994.

5. Bazley LA and Gullick WJ: The epidermal growth factor receptor family. Endocr Relat Cancer 12: S17-S27, 2005.

6. Normanno N, Bianco C, Strizzi L, et al: The ErbB receptors and their ligands in cancer: an overview. Curr Drug Targets 6: 243-257, 2005.

7. Arnold D and Seufferlein T: Targeted treatments in colorectal cancer: state of the art and future perspectives. Gut 59: 838-858, 2001.

8. Bonner JA, Harari PM, Giralt J, et al: Radiotherapy plus cetuximab for squamous-cell carcinoma of the head and neck. N Engl J Med 354: 567-578, 2006.

9. Pao W and Chmielecki J: Rational, biologically based treatment of EGFR-mutant non-small-cell lung cancer. Nat Rev Cancer 10: 760-774, 2010.

10. Gao YS, Hubbert CC and Yao TP: The microtubule-associated histone deacetylase 6 (HDAC6) regulates epidermal growth factor receptor (EGFR) endocytic trafficking and degradation. J Biol Chem 285: 11219-11226, 2010.

11. Deribe YL, Wild P, Chandrashaker A, et al: Regulation of epidermal growth factor receptor trafficking by lysine deacetylase HDAC6. Sci Signal 2: ra84, 2009.

12. Devesa SS, Blot WJ and Fraumeni JF Jr: Changing patterns in the incidence of esophageal and gastric carcinoma in the United States. Cancer 83: 2049-2053, 1998.

13. Gebski V, Burmeister B, Smithers BM, Foo K, Zalcberg J and Simes J: Survival benefits from neoadjuvant chemoradiotherapy or chemotherapy in oesophageal carcinoma: a meta-analysis. Lancet Oncol 8: 226-234, 2007.

14. Jankowski J HD, Hopwood D and Wormsley KG: Expression of epidermal growth factor, transforming growth factor alpha and their receptor in gastro-oesophageal diseases. Dig Dis 11: 1-11, 1993.

15. Gibault L, Metges JP, Conan-Charlet V, et al: Diffuse EGFR staining is associated with reduced overall survival in locally advanced oesophageal squamous cell cancer. Br J Cancer 93: 107-115, 2005.

16. Kim LW, Tsung-Teh W, In Seon C, et al: Expression of epidermal growth factor receptor in esophageal and esophagogastric junction adenocarcinomas. Cancer 109: 658-667, 2007.

17. Wei Q, Chen L, Sheng L, Nordgren H, Wester K and Carlsson J: EGFR, HER2 and HER3 expression in esophageal primary tumours and corresponding metastases. Int J Oncol 31: 493-499, 2007.

18. Oku K, Tanaka A, Yamanishi H, et al: Effects of various growth factors on growth of a cloned human esophageal squamous cancer cell line in a protein-free medium. Anticancer Res 11: 1591-1595, 1991.

19. Ahmed SA, Gogal RM Jr and Walsh JE: A new rapid and simple non-radioactive assay to monitor and determine the proliferation of lymphocytes: an alternative to $\left[{ }^{3} \mathrm{H}\right]$ thymidine incorporation assay. J Immunol Methods 170: 211-224, 1994.

20. Nociari MM, Shalev A, Benias P and Russo C: A novel one-step, highly sensitive fluorometric assay to evaluate cell-mediated cytotoxicity. J Immunol Methods 213: 157-167, 1998.

21. Lennartsson J, Wardega P, Engstrom U, Hellman U and Heldin $\mathrm{CH}$ : Alix facilitates the interaction between $\mathrm{c}-\mathrm{Cbl}$ and platelet-derived growth factor beta-receptor and thereby modulates receptor down-regulation. J Biol Chem 281: 39152-39158, 2006.

22. McLeod HL, Kearns CM, Kuhn JG and Bruno R: Evaluation of the linearity of docetaxel pharmacokinetics. Cancer Chemother Pharmacol 42: 155-159, 1998.

23. Sethi VS, Jackson DV Jr, White DR, et al: Pharmacokinetics of vincristine sulfate in adult cancer patients. Cancer Res 41: 3551-3555, 1981. 
24. Nemoto T, Ohashi K, Akashi T, Johnson JD and Hirokawa K Overexpression of protein tyrosine kinases in human esophageal cancer. Pathobiology 65: 195-203, 1997.

25. Sines T, Granot-Attas S, Weisman-Welcher S and Elson A Association of tyrosine phosphatase epsilon with microtubules inhibits phosphatase activity and is regulated by the epidermal growth factor receptor. Mol Cell Biol 27: 7102-7112, 2007.

26. Flint AJ, Tiganis T, Barford D and Tonks NK: Development of 'substrate-trapping' mutants to identify physiological substrates of protein tyrosine phosphatases. Proc Natl Acad Sci USA 94: 1680-1685, 1997.

27. Eden ER, White IJ, Tsapara A and Futter CE: Membrane contacts between endosomes and ER provide sites for PTP1B-epidermal growth factor receptor interaction. Nat Cell Biol 12: 267-272.

28. Poruchynsky MS, Wang EE, Rudin CM, Blagosklonny MV and Fojo T: Bcl-xL is phosphorylated in malignant cells following microtubule disruption. Cancer Res 58: 3331-3338, 1998.

29. Blagosklonny MV, Giannakakou P, el-Deiry WS, et al: Raf-1/bcl-2 phosphorylation: a step from microtubule damage to cell death. Cancer Res 57: 130-135, 1997.

30. Tarcic G, Boguslavsky SK, Wakim J, et al: An unbiased screen identifies DEP-1 tumor suppressor as a phosphatase controlling EGFR endocytosis. Curr Biol 19: 1788-1798, 2009.

31. Xu Y, Tan L-J, Grachtchouk V, Voorhees JJ and Fisher GJ: Receptor-type protein-tyrosine phosphatase-kappa regulates epidermal growth factor receptor function. J Biol Chem 280: 42694-42700, 2005.
32. Suarez Pestana E, Tenev T, Gross S, Stoyanov B, Ogata M and Bohmer FD: The transmembrane protein tyrosine phosphatase RPTPsigma modulates signaling of the epidermal growth factor receptor in A431 cells. Oncogene 18: 40694079, 1999.

33. Keilhack H, Tenev T, Nyakatura E, et al: Phosphotyrosine 1173 mediates binding of the protein-tyrosine phosphatase SHP-1 to the epidermal growth factor receptor and attenuation of receptor signaling. J Biol Chem 273: 24839-24846, 1998.

34. Agazie YM and Hayman MJ: Development of an efficient 'substrate-trapping' mutant of Src homology phosphotyrosine phosphatase 2 and identification of the epidermal growth factor receptor, Gab1, and three other proteins as target substrates. J Biol Chem 278: 13952-13958, 2003.

35. Yuan T, Wang Y, Zhao ZJ and Gu H: Protein-tyrosine phosphatase PTPN9 negatively regulates ErbB2 and epidermal growth factor receptor signaling in breast cancer cells. J Biol Chem 285: 14861-14870, 2010.

36. Ku GY and Ilson DH: Esophagogastric cancer: targeted agents. Cancer Treat Rev 36: 235-248, 2010.

37. Ekman S, Dreilich M, Lennartsson J, et al: Esophageal cancer: current and emerging therapy modalities. Expert Rev Anticancer Ther 8: 1433-1448, 2008. 